\title{
Frege's Equivalence Thesis and Reference Failure
}

\author{
Nathan Hawkins*
}

Received: 25 February 2020 / Accepted: 20 November 2020

Abstract: Frege claims that sentences of the form ' $A$ ' are equivalent to sentences of the form 'it is true that $A$ ' (The Equivalence Thesis). Frege also says that there are fictional names that fail to refer, and that sentences featuring fictional names fail to refer as a result. The thoughts such sentences express, Frege says, are also fictional, and neither true nor false. Michael Dummett argues that these claims are inconsistent. But his argument requires clarification, since there are two ways The Equivalence Thesis has been formulated, according as the thesis equates the senses or the referents of the relevant sentences. I have two aims in this paper. The first is to demonstrate that a sameness of sense thesis is inconsistent with Frege's other theses. The second is to argue that a sameness of reference thesis is consistent with them. Thus, all else being equal, Frege ought to endorse a sameness of reference, rather than a sameness of sense thesis.

Keywords: Dummett; equivalence; fiction; Frege; true; reference.

* Cambridge University

https://orcid.org/0000-0003-3565-8999

Gonville and Caius College, Cambridge University Trinity St, Cambridge, United Kingdom, CB2 1TA.

\neh37@cam.ac.uk

() The Author. Journal compilation (C) The Editorial Board, Organon F.

This article is distributed under the terms of the Creative Commons Attribution-NonCommercial 4.0 International Public License (CC BY-NC 4.0). 


\section{Introduction}

Frege claims that sentences of the form ' $A$ ' are equivalent to sentences of the form 'it is true that $A$ '. I shall call this claim 'The Equivalence Thesis'. ${ }^{1}$ Frege also says that there are names that fail to refer, and that sentences featuring these names fail to refer as a result. Names and sentences that do not refer he calls 'fictional', as many of his examples are drawn from fiction. Fictional sentences, Frege says, express fictional (or mock) thoughts, which are neither true nor false.

Michael Dummett argues that The Equivalence Thesis is inconsistent with what Frege says about fictional thoughts. But his argument requires clarification, since there are two ways The Equivalence Thesis has been formulated, based on whether the thesis equates the senses or the referents of the relevant sentences. Dummett formulates the thesis as an identity of reference. But a close reading demonstrates that Frege intends to put forward a sameness of sense thesis.

I have two aims in this paper. The first is to demonstrate that a sameness of sense thesis is indeed inconsistent with Frege's theses on fictional sentences and thoughts. This shows that Frege was inconsistent in his claims. The second is to argue that a sameness of reference thesis is consistent with them. Thus, all else being equal, Frege ought to endorse a sameness of reference, rather than a sameness of sense thesis.

The paper is structured as follows: $§ 1$ presents Frege's theses on fictional sentences and fictional thoughts. $§ 2$ presents two versions of The Equivalence Thesis. $\S 3$ argues that Frege's claims about fictional sentences and thoughts are incompatible with the identity of sense version of The Equivalence Thesis. I further argue that there is no way to resolve the conflict. $\S 4$ introduces a non-standard analysis of 'it is true that $A$ ' proposed in the literature and used to defend Frege. I argue that this analysis is also incompatible with Frege's thesis regarding fictional thoughts. $\$ 5$ argues that the sameness of reference thesis is consistent with Frege views about fictional

1 This is the name given to it by Dummett. The same claim is alternately known as 'The Redundancy Thesis', e.g. (Schantz 1998), and 'The Transparency Thesis', e.g. (Kalderon 1997). 
sentences, and $\S 6$ argues the same regarding Frege's views about fictional thoughts. $\S 7$ briefly recaps and concludes.

\section{Frege's theses on reference failure}

Frege's theses on reference failure each emerge from his claim that there are names that do not refer. Such names appear in fiction. For example: 'Frodo' or 'Anna Karenina'. But he also realises that names that do not refer can appear outside of fiction too. For example: 'Vulcan' (Le Verrier's putative planet) or 'the greatest prime number'. Nevertheless, Frege calls all names that do not refer 'fictional', and I shall follow suit.

By the time Frege discusses fictional names, he has a two-level semantics: sense and reference. The reference of a name is, roughly, the object a person thinks about when hearing the name. The sense of a name is, roughly, the way in which a person thinks about the object referred to when hearing the name. If a name refers, it does so by way of its sense $(\mathrm{CP}, 158$; PW, 124) ${ }^{2}$. Since things can be thought about in multiple ways, multiple senses can be associated with the same reference. Frege gives a now-famous example. 'The Morning Star' and 'The Evening Star' have the same reference: Venus. But the senses of the two names differ, for they each express a different way of thinking about Venus.

Frege says fictional names obviously have a sense, otherwise they would be empty sounds and it would be wrong to call them names (CP, 162; PW, 124). Presumably, we think something upon hearing a fictional name. After all, a fictional name 'behaves as if it names' something (PW, 122). But it equally seems clear to Frege that there is no object that such names refer to (CP, 162-3). So fictional names have a sense, but they lack a reference. Frege's focus when speaking of reference failure is names. Names refer (if at all) to objects. But there are also concept-words (PW, 124). For example: 'is a horse' or 'is a planet'. Concept-words refer (if at all) to concepts; what we might now call a 'property'. Frege thinks there are concept-words that have a sense but fail to refer. A concept-word fails to refer if the concept it

2 I will regularly be referring to Frege's works and use a shorthand notation for this purpose. The key for this shorthand can be found in the references section. 
purports to refer to does not have sharp boundaries (PW, 122). A concept has sharp boundaries iff every object either falls or does not fall under it. ${ }^{3}$ Not all purported concepts have sharp boundaries. For example: the concept of being a heap (CN, 177). Concept-words that fail to refer I shall also call 'fictional'.

The distinction between sense and reference applies to declarative sentences as well. (Henceforth the qualification 'declarative' will be dropped.) The sense expressed by a sentence is of a special kind. It is a thought. A sentence expresses a thought that is composed of the senses of its elements. (I use the term 'element' to describe a unit into which a sentence may be analysed.) So the sense of a name or concept-word is part of any thought expressed by sentences in which they appear (CP, 390). For example: the thought expressed by 'Frodo is short' is composed of the sense of 'Frodo' and the sense of 'is short'.

Just as sentences express a special kind of sense, they refer to a special kind of object: a truth-value. There are just two truth-values: the True and the False. Frege thinks that, just as the thought a sentence expresses is determined by the senses of its elements, the truth-value a sentence refers to is determined by the references of its elements. For example: whether 'Venus is a planet' refers to the True or the False is determined by the referent of 'Venus' and the referent of 'is a planet'.

Frege further believes that if a name or concept-word fails to refer, sentences in which they are an element fail to refer also. So if a fictional name is an element in a sentence, the sentence expresses a thought, but does not refer. The sentence itself is fictional. The passage in which Frege most clearly puts this thesis forward is the following:

A sentence containing a non-referring proper name is neither true nor false; if it expresses a thought at all, then that thought belongs to fiction. In that case the sentence has no reference... If a sentence can be split up into parts, each of which has a reference, then the sentence also has a reference. (PW, 194; cf. CP, 162-69).

3 Exception: If the concept under consideration is a higher-level concept, then the matter of sharp boundaries concerns whether concepts one level lower fall under it (CP, 137-46). 
This thesis features heavily in what follows and deserves a name. I call it 'The Contagion Thesis'. ${ }^{4}$ Reference failure is contagious. A proper name or concept-word that fails to refer 'infects' all sentences in which it is an element, and they too fail to refer. Conversely, if all sentential elements refer, the sentence itself refers. I formulate this thesis using a logical schema, where ' $A$ ' (hereafter) is any sentence:

Contagion $_{R}$ ' $A$ ' refers iff every element of ' $A$ ' refers.

The passage also says that sentences that fail to refer express thoughts that 'belong to fiction' (cf. PW, 233). So there is also a form of contagion surrounding the concept of being fictional:

Contagion $_{F}$ ' ' $A$ ' is fictional iff some element of ' $A$ ' is fictional, and ' $A$ ' is fictional iff the thought expressed by ' $A$ ' is fictional.

Sentences, names, and concept-words either refer or are fictional. So Contagion $_{R}$ and the first conjunct of Contagion $F$ differ only in terminology. A person might also extend the concept of referring to include thoughts iff they are expressed by sentences that refer. After all, Frege thinks that it is senses that determine the referent of a sentence (PW, 124-5). If they did so, and I shall, the second conjunct of Contagion $_{F}$ would be a terminological variant of " $A$ " refers iff the thought that $A$ refers', which could then conjoin to Contagion $_{R}$ to provide a terminological variant of Contagion $F$. Because of this, I treat Contagion $R$ and Contagion $F$ as the same thesis, and label it 'Contagion'.

Now, what distinguishes fictional thoughts from other thoughts is that they are neither true nor false. All other thoughts are either true or false tertium non datur (CP, 373; PW, 186, 194).

The claim that fictional thoughts are neither true nor false is bound up with the reference failure of sentences expressing them. For a thought is true iff the sentence expressing it refers to the True. And a thought is false iff the sentence expressing it refers to the False. (Just as I have extended the concept of 'referring to the True' or 'referring to the False' to thoughts expressed by sentences that refer to the True or the False, I will extend the

4 This name did not originate with me. It was suggested to me by Alex Oliver and is based on the description of the thesis in Plural Logic (Oliver \& Smiley 2016, 86). 
concept of being true or being false to sentences that express thoughts that are true or false, as does Frege (CP, 393 fn. 22; PW, 233).) So, if a sentence fails to refer, the thought it expresses is neither true nor false (PW, 194, 233). I call this thesis, 'The Gap Thesis':

Gap: If ' $A$ ' is fictional, then the thought expressed by ' $A$ ' is neither true nor false.

The final thing to say about Frege's views on fictional thoughts concerns judgement. In a judgement, a person acknowledges that a thought is true (CP, 355-6). A judgement that rejects a thought as false is, Frege says, really a judgement that the contradictory thought is true. The contradictory thought is that expressed by the negation of the expressing sentence (CP, 381-5; PW, 198). In the case of fictional thoughts, both the fictional thought and its contradictory are neither true nor false. For example, the thought expressed by 'Frodo is short' is fictional because 'Frodo' is a fictional name. This thought is neither true nor false. The same applies to the contradictory thought: that expressed by 'Frodo is not short'. Since neither a fictional thought nor its contradictory is true, fictional thoughts cannot correctly be judged (CP, 373). The qualification 'correctly' here is important. Frege does not think it is impossible to judge fictional thoughts. Only that anyone who does, mistakenly takes all elements of the sentence to refer (cf. CP, 162; PW, 2). ${ }^{5}$

Since fictional thoughts cannot correctly be judged, they ought not to be used as a means of scientific investigation. Fictional thoughts are neither true nor false. And science is only interested in judging truth and falsity. This does not mean science ought to have no interest in fictional thoughts. For Frege distinguishes between judging fictional thoughts and judgements about fictional thoughts:

'Scylla had six dragon gullets'. This proposition too is neither true nor false but fiction, since the proper name 'Scylla' designates nothing. Such propositions can be the object of a scientific

\footnotetext{
5 When engaged in Fregean exegesis, labelling incorrect judgements 'judgements' is controversial. See, for example, (Kremer 2000) and (Ricketts 1986). The issue is irrelevant to the argument I make in this paper.
} 
examination, e.g., one concerned with mythology, but no scientific investigation can be carried out using them. (BLA, II 76).

Here Frege makes a distinction between a thought as an object of investigation and a thought as a vehicle of investigation. When a thought is an object of investigation, judgements are made about which concepts that thought falls under; concepts such as the concept of being believed to be so. When a thought is the vehicle of investigation, judgements are made about the referents of the senses that constitute the thought. Consider, for example, the thought expressed by 'Cleopatra was beautiful'. This thought would be an object of investigation when judging whether historians believe that Cleopatra was beautiful. But it would be a vehicle of investigation when judging whether the object referred to by 'Cleopatra' fell under the concept referred to by 'was beautiful', i.e. when judging whether Cleopatra was beautiful. In the case of fictional thoughts, Frege says they ought to only be objects of investigation, not vehicles. So while it is not possible to correctly judge whether Vulcan orbits the Sun, it is possible to correctly judge whether Le Verrier believed that Vulcan orbits the Sun.

Judgements about fictional thoughts, then, are much the same as judgements about any object. Fictional thoughts are part of the furniture about which science makes judgements. To make an assertion about a thought, fictional or otherwise, the thought must be referred to and then predicated of. To refer to a thought, a person can use the word 'that'. According to Frege, 'that' indicates an indirect context for the sentence that follows it. In indirect contexts, sentences refer to the thought they ordinarily-in $d i$ rect contexts - express (CP, 159). So while 'Frodo is short' is a sentence that expresses a fictional thought but lacks reference, 'that Frodo is short' is a name that refers to the fictional thought expressed by 'Frodo is short' and has a sense which is a way of thinking about this thought (CP, 166). Fictional sentences, fictional names and fictional concept-words are also things about which judgements can be made, for they may each be referred to by using quotation marks (CP, 159).

Now, I will be making rather heavy use of indirect contexts in what follows. Using the 'that' idiom for these purposes becomes unwieldy when attempting more complicated analysis. For while quotation marks provide a convenient way to mark the scope of the context change by providing a 
start and end point, the word 'that' does not clearly mark its scope. This is especially unhelpful when indirect contexts become nested, such as in the sentence: 'that that Frodo is short is fictional is true'. Because of this, I will use angled brackets as indirect context indicators that function in a similar way to quotation marks. For example, the angled brackets notation can be used to turn the previous example into the much clearer: ' $<<$ Frodo is short $>$ is fictional $>$ is true'. In this notation, ' $<$ Frodo is short $>$ ' can be read as 'that Frodo is short'.

I conclude this section by summarising the discussion about judgement above, making the role of context clear. 'Frodo is short' cannot be correctly asserted; the thought it expresses is fictional and cannot be correctly judged. But ' $<$ Frodo is short $>$ ' and "Frodo is short"' are not fictional; they refer to a fictional thought and a fictional sentence respectively. Since these names refer, assertions can be made about their referents. Importantly, these assertions include instances of Gap, e.g. "if "Frodo is short" is fictional, then $<$ Frodo is short $>$ is neither true nor false', and instances of Contagion, e.g. "Frodo is short" refers iff every element of "Frodo is short" refers'.

\section{The Equivalence Thesis}

There is some confusion regarding the content of what I have called 'The Equivalence Thesis'. Frege states it in several places. One is:

The sentence 'I smell the scent of violets' has the same content as 'It is true that I smell the scent of violets'. (CP, 354; cf. CP, 164; PW, 129, 141, 194, 233-4, 251-2).

Frege's discussion here, as elsewhere, mentions a specific case. But the examples he uses vary. The claim is obviously meant to generalise.

In Frege: Philosophy of Language, Dummett (1981, 445) formulates the thesis, immediately prior to his argument that I will discuss, using a biconditional schema:

Equivalence $_{R}:\langle A\rangle$ is true iff $A$.

'Iff' is a material biconditional. A material biconditional is, for Frege, a sign for identity between truth-values, i.e. it is a sign for identity of reference. 
Equivalence $_{R}$ implies, for example, that the sentences 'I smell the scent of violets' and 'it is true that I smell the scent of violets' refer to the same truth-value. So Equivalence $e_{R}$ can also be rendered: $\langle A\rangle$ is true $=A$.

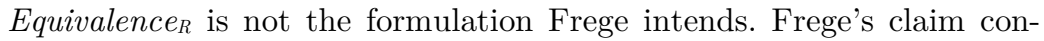
cerns the identity of the expressed thoughts, not the identity of their truthvalues. That Frege is concerned with identity of sense, rather than reference, is made especially clear in another instance in which he describes the thesis:

Again in the two sentences 'Fredrick the Great won the battle of Rossbach' and 'It is true that Fredrick the Great won the battle of Rossbach', we have, as we said earlier, the same thought in a different verbal form. (PW, 141).

Now, Dummett is not ignorant of the fact Frege took his thesis to be about sameness of sense. In an earlier article Truth, where Dummett (1959) presents the same argument, he formulates Frege's thesis as one concerning sense:

Equivalences: $<<A>$ is true $>=<A>$.

The difference in formulation Dummett provides in these places is liable to confuse. With the likely result being that Dummett's conclusion is thought (presumably by Dummett also) to hold against both formulations.

Adding to this potential for confusion is the fact that the formulations are believed to be closely related. For example, Crispin Wright (1998) describes Equivalences and Equivalencer as 'tantamount' to each other (66, cf. 60). But they are not tantamount at all. Reference does not determine sense. Since identity of reference is distinct from identity of sense, a person could claim there is a general identity of reference between sentences of this form (accept Equivalence $e_{R}$ ), but that in each case they express distinct thoughts (reject Equivalences). This is a point made by Wolfgang Künne (2003, 35 fn. 8). Yet Künne thinks the reverse implication holds: 'if you accept an instance of [Equivalences] you are committed to endorsing the corresponding instance of [Equivalence $]^{\prime}$ ' (Künne 2003, 35). Presumably, he thinks that this follows from the fact that sense determines reference (PW, 124-5). But he is incorrect. Fictional sentences have a sense but no reference. And in almost all cases in which Frege discusses The Equivalence Thesis, he speaks of fictional contexts too (CP, 164; PW, 194, 234, 251). So 
what is expressed by 'it is true that Frodo is short' is, for Frege, the same as what is expressed by 'Frodo is short'. If ' $A$ ' were 'Frodo is short' then the relevant instance of Equivalences would be true (presuming Equivalences), while the relevant instance of Equivalencer would not refer, and so be neither true nor false. Thus, Equivalences and Equivalence $e_{R}$ are distinct theses, and must be kept separate. Neither is a strictly stronger or weaker version of the other.

\section{The incompatibility of equivalences with contagion and gap}

In this section I demonstrate the incompatibility of Equivalences with Contagion and Gap. The incompatibility is two-fold. There is incompatibility between Equivalences and Contagion, and there is incompatibility between Equivalences and Gap. The latter is the incompatibility described by Dummett, but it is the former that demonstrates the nature of the incompatibility more clearly. I discuss it first.

The incompatibility between Equivalences and Contagion can be shown as follows: Let ' $X$ ' (hereafter) be any fictional sentence. Then ' $X$ ' does not refer. Now consider the sentence ' $\langle X\rangle$ is true'. ' $\langle X\rangle$ is true' has two elements: ' $\langle X\rangle$ ' and 'is true'. It says that an object, $\langle X\rangle$, falls under a concept, the concept of being true. Although ' $X$ ' fails to refer when standing alone, in ' $\langle X\rangle$ is true' the sentence ' $X$ ' appears in an indirect context, and ' $\langle X\rangle$ ' refers to the fictional thought expressed by ' $X$ '. If all elements of ' $\langle X\rangle$ is true' refer, the sentence refers (by Contagion). This means that ' $\langle X>$ is true' is not fictional, i.e. it expresses a thought that refers. In contrast, ' $X$ ' is fictional, i.e. it expresses a fictional thought. Since the thought $\langle X\rangle$ is fictional while the thought $\langle<X\rangle$ is true $\rangle$ is not, they cannot be the same thought by the indiscernibility of identicals. Conclusion: Contagion and Equivalences are incompatible.

There is no way to rescue Equivalence given a surface form analysis of ' $\langle X\rangle$ is true'. Since $\langle X\rangle$ is fictional, any attempt would require $\langle<X>$ is true $>$ to also be fictional. This means ' $\langle X\rangle$ is true' would have to be, despite appearances, a fictional sentence. This leaves only two options. 
First, it might be argued that, even though the elements in ' $\langle X\rangle$ is true' refer, the sentence fails to. This would require weakening the relevant part of Contagion from a biconditional to a conditional: ' $A$ ' refers only if every element of ' $A$ ' refers. Second, a person could argue that there is an element in ' $\langle X\rangle$ is true' that fails to refer. There are only two candidates: ' $\langle X\rangle$ ' and 'is true'. By hypothesis, ' $\langle X>$ ' refers to a fictional thought. This means it would have to be 'is true' that fails to refer.

The first option can be dismissed. For if ' $\langle X\rangle$ is true' fails to refer, it cannot be correctly judged whether $\langle X\rangle$ falls under the concept of being true. So there is no fact of the matter whether this thought falls under the concept of being true (if there were, it could be correctly judged (BLA, I $8)$ ). In that case, since $\langle X\rangle$ is an object, the concept of being true lacks sharp boundaries, and 'is true' fails to refer after all. The first option would then become the second option.

The second option can also be dismissed. If 'is true' did not refer, in cases where ' $A$ ' is not a fictional sentence, ' $A$ ' would refer, while ' $\angle A>$ is true', since it contains 'is true' as an element, would not refer. Hence $<A>$ would not be a fictional thought, while $<<A>$ is true $>$ would be a fictional thought. Since these thoughts are distinct, Equivalences has false instances. Only this time it is non-fictional sentences that produce false instances, rather than fictional ones.

Although I have demonstrated that Equivalences is incompatible with Contagion, it is still worthwhile examining Dummett's argument for its incompatibility with Gap, especially since Dummett aims his argument at Equivalence $_{R}$. His argument is as follows:

Suppose that $A$ is a sentence which expresses a thought which may, in certain circumstances, be neither true nor false. Then the sentence 'It is true that $A$ ' cannot be equivalent to $A$ : for, when the thought expressed by $A$ is neither true nor false, say because $A$ contains a name which has a sense but lacks a bearer, the thought expressed by 'It is true that $A$ ' will be false, although, by hypothesis, that expressed by $A$ is not false. (Dummett 1981, 445; cf. Dummett 1959, 145-6).

Dummett's conclusion, as it stands, says that $\langle X>$ and $\langle<X>$ is true $>$ cannot be identical due to the law of indiscernibility of identicals (one being 
false but not the other). Thus Equivalences has false instances given Gap. This is clearer when the argument is formulated step by step with an example:

1: $<$ Frodo is short $>$ is not true and $<$ Frodo is short $>$ is not false. ( Gap instance consequent.)

2: $<$ Frodo is short $>$ is not true. (From 1.)

3: $<<$ Frodo is short $>$ is true $>$ is false. (From 2.)

4: $<$ Frodo is short $>$ is not false. (From 1.)

Conclusion: $<$ Frodo is short $>$ and $<<$ Frodo is short $>$ is true $>$ are not identical. (From 4, 3, and the indiscernibility of identicals.)

The argument succeeds, then, against Equivalences. But at the start of this quotation, Dummett says, "the sentence "It is true that $A$ " cannot be equivalent to $A$ ', which, given that he formulates The Equivalence Thesis with Equivalencer, suggests that an equivalence of reference thesis also has false instances. I return to Dummett's argument in $\S 6$ to see how it might be adapted to apply to Equivalence $e_{R}$ and show why any attempt to do so fails.

\section{Rescue by alternative analysis?}

In the previous section I have demonstrated the incompatibility of Equivalences with Contagion and Gap, given an analysis of ' $\langle A\rangle$ is true' according to surface form. But several exegetes believe Frege thought the sentence should not be analysed this way for reasons independent of Dummett's argument. Paul Horwich (2010), for example, says that by endorsing Equivalences $_{S}$ Frege implies that the logical form of ' $\langle A\rangle$ is true' is 'not what it would seem to be: i.e. not " $X$ is $F$ "' (39). (See also (Burge 1986; Davidson 1969; Grover, Camp, \& Belnap 1975) for other analyses of ' $<A>$ is true' that differ from surface-form.) Some exegetical justification for ascribing to Frege an alternative analysis comes from what he says about the deceptive nature of the grammar of the sentence ' $\angle A>$ is true':

If we say 'the thought is true' we seem to be ascribing truth to the thought as a property. If that were so, we should have a case 
of subsumption. The thought as an object would be subsumed under the concept of the true. But here we are misled by language. We don't have the relation of an object to a property, but that of the sense of a sign to its referent. (PW: 194; cf. CP: 164, 3545 ; my emphasis)

Although the other analyses on offer differ in detail, they all fall under the umbrella of what Künne (2003, 34-5) calls a 'truth-frame' analysis. The truth-frame is the words 'that... is true' or 'it is true that...'. Or in my notation: ' $<\ldots>$ is true'. Frege's thesis, according to Künne and others like him, says that if a person replaces ' $A$ ' in 'that $A$ is true' with a sentence, the thought expressed is identical to that expressed by the inserted sentence (Künne 2008, 14). We might think of it this way: rather than analysing 'that $A$ is true' as a case in which 'that' indicates an indirect context for ' $A$ ' such that it refers to the thought it expresses in direct contexts, and then predicates 'is true' of this thought; a person instead takes the sentence ' $A$ ' and inserts it into the frame 'that... is true' to produce another sentence. The truth-frame cannot be further analysed into 'that' and 'is true'. It must be taken as a whole. A whole that has no effect on the sense of the sentence inserted into it (cf. PW, 251-2). Hence in ' $\angle A>$ is true', according to the truth-frame analysis, 'is true' is not a concept-word, and the 'that' in 'that $A$ is true' does not indicate a change of context. The context for ' $A$ ' in ' $\angle A>$ is true' is direct rather than indirect. Under such an analysis, as Davidson $(1969,749)$ says, it is only a 'freak of grammar' that 'that $A$ is true' consists of a complex singular term and a predicate.

My purpose in this paper is not to adjudicate between analyses of ' $<A>$ is true'. It is only to examine whether some version of The Equivalence Thesis is compatible with Frege's theses about fictional sentences and thoughts. If it is credible to think Frege took ' $\angle A>$ is true' to have a nonstandard analysis, and if that non-standard analysis resolves the incompatibility, then, all else being equal, this would seem to be the most charitable way to interpret him. However, as I will show in this section, the incompatibility remains regardless of analysis.

Now, the truth-frame analysis does resolve the incompatibility of Equivalences with Contagion. For in the truth-frame analysis, the context of ' $X$ ' in ' $\langle X\rangle$ is true' is direct, not indirect. So the elements of ' $X$ ' are also 
elements of ' $\langle X\rangle$ is true'. This means the incompatibility is avoided. For suppose ' $X$ ' were 'Frodo is short'. 'Frodo is short' fails to refer because one of its elements, 'Frodo', fails to refer. And this element is also (given the truth-frame analysis) an element of ' $<$ Frodo is short $>$ is true'. So ' $<$ Frodo is short $>$ is true', like 'Frodo is short', fails to refer by Contagion. Since the thoughts expressed by both sentences are fictional, they could be identical, and thus the pair of sentences does not necessarily constitute a false instance of Equivalences. This observation generalises to all instances where ' $A$ ' is fictional since all fictional sentences contain a fictional element (by Contagion).

As regards the incompatibility between Equivalences and Gap, Künne (2003, 37-42) argues that under the 'correct' truth-frame analysis, no incompatibility of the kind Dummett describes arises. Presumably Künne therefore thinks there is no incompatibility at all. But, given a truth-frame analysis, a new problem arises that Künne does not address. The problem is that Equivalences, given a truth-frame analysis, means Gap is unable to say anything true about fictional thoughts. So although Equivalences coupled with Gap does not lead to contradiction, it is incompatible with the intent of Gap. For, given a truth-frame analysis of ' $\angle A>$ is true', Gap is unable to assert anything about fictional thoughts, the reason for which Frege stated it.

This point is best demonstrated by example. Under the truth-frame analysis, ' $<$ Frodo is short $>$ is true' is fictional. The reason it is fictional, so the analysis goes, is because the context of 'Frodo is short' in the sentence is direct, and so 'Frodo' is an element of the sentence. But then, 'Frodo' must also be an element of ' $<$ Frodo is short $>$ is not true'. This sentence is simply the negation of the previous one, and negation does not affect context. So ' $<$ Frodo is short $>$ is not true' is also a fictional sentence. Since ' $<$ Frodo is short $>$ is not true' is an element of ' $<$ Frodo is short $>$ is not true and not false', this sentence must also be fictional. In fact, nothing about the above relied on the particular example, so, given the truth-frame analysis, ' $\angle A>$ is not true and not false' is fictional if ' $A$ ' is. Now ' $\angle A>$ is not true and not false', for some ' $A$ ', is an element of all instances of Gap. So, under a truth-frame analysis, any instance of Gap in which ' $A$ ' is fictional will be fictional rather than true. This means Gap could never 
correctly assert that a fictional thought is neither true nor false. Although this is not a contradiction, I take it to be an incompatibility.

Note also that the problem just highlighted is independent of whether Equivalences or Equivalencer is the way The Equivalence Thesis is formulated. It is a problem regarding the alternative analyses applied directly to Gap. So the alternative analyses cannot be of use in resolving the incompatibility, regardless of which version of The Equivalence Thesis is under consideration.

\section{The compatibility of Equivalence ${ }_{R}$ with Contagion}

In §3 I showed that, when ' $A$ ' is fictional, the relevant instance of Equivalences is incompatible with both Contagion and Gap given a surface-form analysis of ' $<A>$ is true'. In $\S 4$ I showed that alternative analyses are no help. So if The Equivalence Thesis, Contagion and Gap are to be compatible, The Equivalence Thesis could only be formulated by Equivalence $e_{R}$ and ' $<A>$ is true' given a surface-form analysis. This section argues that, under these circumstances, The Equivalence Thesis is compatible with Contagion. The following section will argue it is also compatible with Gap.

Contagion says that a sentence refers iff all its elements refer. Consider 'Frodo is short'. This sentence does not refer, because 'Frodo', an element of it, does not refer. Now consider ' $<$ Frodo is short $>$ is true'. This sentence refers, because its only elements are ' $<$ Frodo is short $>$ ' and 'is true', both of which refer. So, by Contagion, we can say both:

C1: 'Frodo is short' fails to refer and

C2: ' $<$ Frodo is short $>$ is true' refers.

To see what can be concluded from $C 1$ and $C 2$, we need to do some analysis. All elements of $C 1$ and $C 2$ refer. "Frodo is short"" and " $<$ Frodo is short $>$ is true"' refer to the sentences 'Frodo is short' and '<Frodo is short $>$ is true' respectively, 'fails to refer' refers to the concept of not referring, and 'refers' refers to the concept of referring. Because all elements of $C 1$ and C2 refer, they express thoughts that refer, by hypothesis, to the True. So these thoughts can be correctly judged, and the sentences $C 1$ and C2 can be correctly asserted. What $C 1$ asserts is that the sentence 'Frodo 
is short' falls under the concept of not referring, while C2 asserts that the sentence ' $<$ Frodo is short $>$ is true' falls under the concept of referring. By applying the indiscernibility of identicals to the claims of $C 1$ and $C 2$ we can conclude that the sentences 'Frodo is short' and ' $<$ Frodo is short $>$ is true' are not identical. But this conclusion is irrelevant since it was never in doubt. The relevant question is whether we can further conclude from $C 1$ and $C 2$ that Equivalence E $_{R}$ has false instances.

Now, it seems as though $C 1$ and $C 2$ are incompatible with Equivalencer.

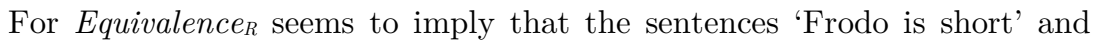
' $<$ Frodo is short $>$ is true' co-refer. Since one sentence refers while the other does not, they cannot co-refer. But the case is not so simple. Equivalence contains no elements that refer to sentences. So it does not imply anything about sentences and whether and to what they refer. The instance of Equivalence $_{R}$ that seems to be incompatible with $C 1$ and $C 2$ is:

$E:<$ Frodo is short $>$ is true $=$ Frodo is short .

By Contagion, $E$ does not refer, for 'Frodo' is an element of $E$ (on the right-hand side), and 'Frodo' does not refer. So, given $C 1$ and $C 2$ or otherwise, $E$ is not false but fictional. This means that the thought expressed by $E$ is fictional and cannot correctly be judged, so neither $E$ nor its negation can be correctly asserted, unlike $C 1$ and $C$ 2. The same would be

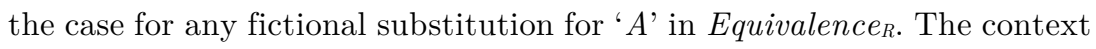

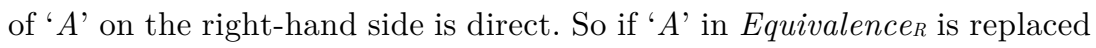
by a fictional sentence, the result is a fictional instance. In other cases, where ' $A$ ' in Equivalence E is replaced by a referring sentence, the result is a true instance. For if ' $A$ ' is false, both ' $\angle A>$ is true' and ' $A$ ' are false, so the instance is true. And if ' $A$ ' is true, both ' $\langle A\rangle$ is true' and ' $A$ ' are true, so the instance is true. So then, Equivalence $e_{R}$ has true instances and fictional instances, but no false ones. The question to be answered, then, concerns what to do about instances of logical schema that are fictional.

Before turning to the case at hand, that of Equivalence $e_{R}$, let me first point out that the question is more general than might be supposed. For example, the self-identity of objects is often expressed using the schema: $a=a$. Frege expresses it this way himself. In fact, he not only endorses 
' $a=a$ ', he says that it holds a priori (CP, 157). Now, ' $a=a$ ' could hold a priori only under the proviso that ' $a$ ' refers. If ' $a$ ' does not refer, ' $a=$ $a$ ' is fictional, not true. For example, it is not true that Frodo $=$ Frodo, just as it is not true that Dr Jekyll = Mr Hyde. And whether ' $a$ ' refers is only sometimes determinable a priori. For example, whether 'the greatest prime number' refers can, for Frege, be determined a priori, but whether 'Vulcan' refers can only be determined a posteriori (cf. FOA, 3-4). So then, ' $a=a$ ' is only a priori true, as Frege claims, if ' $a$ ' can only be substituted for referring names.

Note further that Equivalences is not immune from proviso either. For if ' $A$ ' were substituted by a senseless sentence, say 'the mome raths outgrabe', Equivalences would also have fictional instances. For in this case '<the mome raths outgrabe $>$ ' on the right-hand side of the instance would not refer because the sentence lacked sense. This to say the logical schema Equivalences also has fictional instances unless a proviso is adopted such that only senseful sentences may be substituted for ' $A$ '.

Now, Frege is very aware that non-referring terms (and, by extension, senseless terms) cause logical difficulties. Difficulties that arise from the fact the law of excluded middle fails to hold concerning fictional thoughts (cf. $\mathrm{PW}, 155)$. He points to occasions 'even in mathematics' where signs that fail to refer have led to 'errors' (CP, 169). Hence he says:

A logically perfect language should satisfy the conditions, that every expression grammatically well constructed as a proper name out of signs already introduced shall in fact designate an object, and that no new sign shall be introduced as a proper name without being secured a referent. (CP, 169).

This is why Frege is at pains to secure both sense and reference for the primitive terms in his Conceptual Notation (BLA, I 50). And he makes it a principle that any other terms introduced by definition must also refer (BLA, I 45, I 51).

Of course, natural language is not logically perfect. There are many names that occur without reference. This is not a failing of natural language per se, for without it we would not be able to craft fictional characters. It is however, for Frege, a logical failing. If natural language were purely logical, every sign would refer. Such is the case with Conceptual Notation, it 
is a language designed for logical purposes, thus Frege is at pains to secure a referent for every term (CN, 104-5).

The schemata we have been examining are not schemata of Conceptual Notation, they are logical schemata of natural language. Nevertheless, Frege implicitly assumes instances of logical schemata, even if they are instances of schemata of natural language, only hold on the presumption that all elements refer (cf. CP, 162-3). They are scientific schemata, and in the realm of science all signs have reference (PW, 232). If the thought expressed by an instance of the schemata is fictional then 'truth in the scientific sense' is not in question, and it must be placed to the side (CP, 373; PW, 186). It would be as scientifically (and logically) inappropriate to inquire whether $<$ Frodo is short $>$ is true $=$ Frodo is short, as it would be to inquire whether the greatest prime number were odd.

This implicit proviso applies to all logical schemata of natural language, whether Equivalences, ' $a=a$ ', or ' $A$ iff $A$ '. All of these schemata have fictional instances when their schematic letters are replaced with fictional names or sentences. And this is why Equivalence is incompatible with $C o n-^{-}$

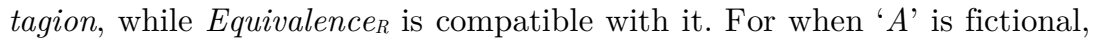

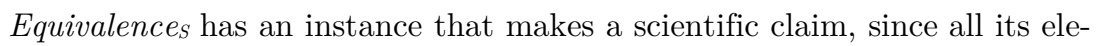
ments refer. This claim is incompatible with the claims of Contagion and can be determined to be so. However, the corresponding instance of Equivalence $_{R}$ makes no scientific claims. It is (scientifically) silent, so is not incompatible with Contagion.

Another way to approach it is to recall Frege's distinction between thoughts as vehicles and thoughts as objects. When ' $A$ ' is fictional the instance of Equivalences refers to the fictional thought expressed by ' $A$ ', making it an object of investigation. Thus a scientific investigation of the thought can proceed, one that runs into contradiction with Contagion. While the corresponding instance of Equivalence $e_{R}$ expresses, rather than refers to, the fictional thought, making it a vehicle of investigation, and thus not amenable to scientific investigation (cf. §1). The upshot is that Equivalence $_{R}$ is compatible with Contagion because it speaks only when it is safe to do so. 


\section{The compatibility of equivalence ${ }_{R}$ with gap}

There appears to be an incompatibility between Equivalence $e_{R}$ and Gap different from that which appeared to occur between Equivalence $e_{R}$ and Contagion. For given the obvious interpretations of the concepts of being true and the concepts of being false, it must be the case that:

Truth: $\langle A>$ is true iff $A$.

Falsity: $\langle A>$ is false iff not $A$.

Truth is, of course, Equivalence $e_{R}$ provided with a name more suited to this section. And Falsity is simply the analogue of it for 'is false'. Note also that Falsity is compatible with Contagion for just the same reasons Equivalence $_{R}$ (i.e. Truth) is. If ' $A$ ' is false, both ' $\angle A>$ is false' and 'not $A$ ' are true, so the instance is true. If ' $A$ ' is true, both ' $\langle A\rangle$ is false' and 'not $A$ ' are false, so the instance is true. And if ' $A$ ' is fictional, Falsity, like Equivalence $_{R}$, is scientifically silent, and thus compatible with Contagion.

Now, Gap says that fictional thoughts fall under neither the concept of being false nor the concept of being true. In this respect, fictional thoughts are just like objects that are not thoughts. The Sun, the number 2, the tea in my teapot, and Frege, are all neither true nor false. This means that the left-hand sides of Truth and Falsity could never be correctly asserted concerning fictional thoughts. In addition, since fictional thoughts cannot be judged, neither ' $X$ ' nor 'not $X$ ' could be correctly asserted. This means the right-hand sides of Truth and Falsity could also never be correctly asserted concerning fictional thoughts. It seems, then, that Truth and Falsity are irrelevant to fictional sentences and thoughts. Hence their silence on the matter.

But suppose that we negate both sides of Truth and Falsity to get:

Truth*: $<A>$ is not true iff not $A$.

Falsity*: $\langle A>$ is not false iff $A$.

From these it appears that we can derive a contradiction from Gap as follows:

1: $\langle X>$ is not true and $\langle X>$ is not false. (From Gap)

2: $\langle X>$ is not true. (From 1 ) 
3: Not $X$. (From 2 via Truth $^{*}$ )

4: $\langle X>$ is not false. (From 1)

5: $X .($ From 4 via Falsity*)

Contradiction: $X$ and Not $X$. (From 3 and 5)

Something puzzling has happened. Each step of the argument appears to be legitimate. There was no obvious contradiction before, and yet a contradiction was derived. Something has gone wrong.

In fact, two things have gone wrong. The first error was that we ought not to perform derivations from or using schemata. For Frege, inferences cannot be made by using schemata but only by using instances of schemata. He explains this in a letter to Hugo Dingler:

We can indeed prove the proposition... 'if $a>b$, then $a+1>b$ $+1 '$ '.. This proposition seems to be of exactly the same kind as the proposition... 'if $3>2$, then $3+1>2+1$ '... and yet the case is entirely different. After we have recognised the proposition ' $3>$ 2 ' as true, we can use it to prove the proposition ' $3+1>2+$ 1 '; but we cannot use the proposition ' $a>b$ ' to prove the proposition ' $a+1>b+1$ '; for ' $a>b$ ' is not a proper proposition because it does not express a thought, nor consequently can it be recognised as true. (PMC, 20-1).

In our case, Truth* cannot license an inference from ' $\langle X\rangle$ is not true' to 'not $X$ ' (step 3), but only from ' $<X>$ is not true' (for a particular ' $X$ ') to 'not $X$ ' (for that same ' $X$ ') via an instance of Truth* (where ' $A$ ' is that ' $X$ '). So we must not assume that the derivations performed above can be used to establish a contradiction. Instead, to show that a contradiction follows from Gap via the schemata, it must be shown that a contradiction can be inferred from a particular instance of it via relevant instances of the schemata.

Let us correct this error now, and restate the slightly longer derivation for a familiar case:

1: $<$ Frodo is short $>$ is not true and $<$ Frodo is short $>$ is not false. (From Gap)

2: $<$ Frodo is short $>$ is not true. (From 1 )

3: $<$ Frodo is short $>$ is not true iff Frodo is not short. (Truth* instance) 
4: Frodo is not short. (From 2 and 3)

5: <Frodo is short $>$ is not false. (From 1)

6: <Frodo is short $>$ is not false iff Frodo is short. (Falsity* instance)

7: Frodo is short. (From 5 and 6)

Contradiction: Frodo is not short and Frodo is short. (From 4 and 7)

The second error was to present an argument that engaged in pseudoinference as if it engaged in inference. Frege describes the distinction between inference and pseudo-inference in an earlier letter to Dingler:

Suppose we have arbitrarily formed the propositions ' $2<1$ ' and 'If something is smaller than 1 , then it is greater than 2 ' without knowing whether these propositions are true. We could derive ' $2>2$ ' from them in a purely formal way; but this would not be an inference because the truth of the premises is lacking. And the truth of the conclusion is no better grounded by means of this pseudo-inference than without it. (PMC, 17; cf. BLA, II 256-7).

Pseudo-inference occurs when sentences are derived from others in a formal way without judgement being made regarding the thoughts they express. It is akin to the notion of logical consequence (cf. Smith 2009). In Fregean terms, we demonstrated that a contradiction can be pseudo-inferred from Gap along with relevant instances of the schemata. In other words, we demonstrated that a contradiction could be derived from them in 'a purely formal way'. Frege sees formal derivations of this sort as assertions of a (single) conditional compound thought:

Before acknowledging its truth, one cannot use a thought as premise of an inference, nor can one infer or conclude anything from it. If anyone still thinks this can be done, he is apparently confusing acknowledgement of the truth of a hypothetical [i.e. conditional] compound thought with performing an inference in which the antecedent of this compound is taken for a premise. (CP, 402-3).

The pseudo-inference argument (conditional compound thought) above, then, ought to be expressed as a single sentence: 
If $<$ Frodo is short $>$ is not true and $<$ Frodo is short $>$ is not false (from Gap)

and $<$ Frodo is short $>$ is not true iff Frodo is not short Truth $^{*}$ instance)

and $<$ Frodo is short $>$ is not false iff Frodo is short (Falsity* instance)

then Frodo is not short and Frodo is short (Contradiction).

The problem is now made plain. This sentence cannot be asserted, for it contains non-referring elements, in particular both cases of 'Frodo' that appear on the right-hand side of each of the instances of Truth* and Falsity*. Thus the pseudo-inference argument we presented is fictional, not scientific. The argument is neither true nor false. In other words, a contradiction does not follow by 'logical consequence' from Gap and Equivalence $e_{R}$. Neither can the argument be re-expressed in terms of inference rather than pseudo-inference for similar reasons. Inference involves judgements. A judgement is the recognition of the truth of a thought. And a judgement is expressed by an assertion. To qualify as an inference to a contradiction, each of the thoughts must be judged. And to express this inference, the premises must be asserted (CN, 117-20; BLA I, 25-6; PMC, 16-7, 78-9). But two of the premises, namely the relevant instance of Truth* and Falsity*, cannot be correctly asserted. And without being asserted, they cannot be used to further assert, say, 'Frodo is not short' on the basis of ' $<$ Frodo is short $>$ is not true'.

These observations generalise to block any attempt to demonstrate an incompatibility between Gap and Equivalence ${ }_{R}$ (Truth). Any 'Dummettian' argument towards a contradiction between Gap and Equivalence $e_{R}$ would have to be of the form we have been examining, and thus involve an incorrect assertion. Instances of Truth, Falsity, Truth* , and Falsity* that can be correctly asserted, i.e. are not fictional, could only be used to infer from Gap such general banalities as:

$<<X>$ is not true $>$ is true and $<<X>$ is not false $>$ is true (via Truth), $<<X>$ is true $>$ is false and $<<X>$ is false $>$ is false (via Falsity), $<<X>$ is true $>$ is not true and $<<X>$ is false $>$ is not true (via Truth $^{*}$ ), and

$<<X>$ is not true $>$ is not false and $<<X>$ is not false $>$ is not false (via Falsity*). 
The second of these is the one that is inconsistent with Equivalences (see $\S 3)$. But none pose a threat to Equivalencer.

\section{Conclusion}

I take myself to have achieved the aims I set forward in this paper. I have shown that, while Equivalences, the equivalence thesis Frege's endorses, is incompatible with Frege's views on fictional sentences and

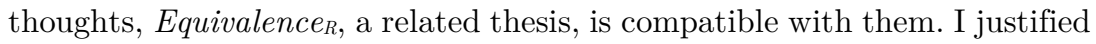
my positive claim by arguing that any attempt to demonstrate an incompatibility between Equivalencer and Frege's theses on fictional sentences and thoughts requires an incorrect assertion. Thus, all else being equal, Frege ought to endorse a sameness of reference, rather than a sameness of sense thesis.

\section{Acknowledgements}

Foremost, I would like to thank my supervisor Prof. Alex Oliver. His close readings of drafts and insightful comments have sharpened my thinking on these topics and improved the way in which I present my position. I am also grateful to the constructive comments I received on my submission to this journal from an anonymous reviewer. I also thank Prof. Michael Potter and Benjamin Marschall for comments on a late draft, and Wouter Cohen and Emma Curran for their useful feedback when I presented this material at a seminar. I would also like to thank the Gates Cambridge Trust for making this research possible.

\section{Funding}

Gates Cambridge Trust.

\section{References}

References to Frege's works:

Frege, Gottlob. 1972. Conceptual Notation and Related Articles. Translated by

Terrell Ward Bynum. Oxford: Oxford Clarendon Press. [CN]

Frege, Gottlob. 1980. Philosophical and Mathematical Correspondence. Translated by Hans Kaal. Oxford: Basil Blackwell. [PMC] 
Frege, Gottlob. 2013. Basic Laws of Arithmetic. Translated by Philip A. Ebert,

Marcus Rossberg, and Crispin Wright. Oxford: Oxford University Press. [BLA]

Frege, Gottlob. 1979. Posthumous Writings. Translated by Peter Long \& Roger

White. Oxford: Basil Blackwell. [PW]

Frege, Gottlob. 1980. The Foundations of Arithmetic. Translated by J. L. Austin.

Oxford: Basil Blackwell. [FOA]

Frege, Gottlob. 1984. Collected Papers on Mathematics, Logic, and Philosophy.

Translated by Max Black, V. H. Dudman, Peter Geach, Hans Kaal, E. H. W.

Kluge, Brian McGuinness, and R. H. Stoothoff. Oxford: Basil Blackwell. [CP]

Other References:

Burge, Tyler. 1986. "Frege on Truth." In Truth, Thought, Reason: Essays on

Frege, 83-132. Oxford: Clarendon Press. https://doi.org/10.1093/acprof.

Davidson, Donald. 1969. "True to the Facts." The Journal of Philosophy 66 (21):

748-64.

Dummett, Michael. 1959. "Truth". Proceedings of the Aristotelian Society 59: 14162.

Dummett, Michael. 1981. Frege Philosophy of Language. London: Duck-worth.

Grover, Dorothy L., Joseph L. Camp, and Nuel D. Belnap Jr. 1975. "A Prosentential Theory of Truth". Philosophical Studies 27 (2): 73-125.

https://doi.org/10.1007/BF01209340.

Horwich, Paul. 2010. Truth - Meaning - Reality. Oxford: Oxford University Press. https://doi.org/10.1093/acprof

Kalderon, Mark Eli. 1997. "The Transparency of Truth". Mind 106 (423): 475-97. https://doi.org/10.1093/mind/106.423.475

Kremer, Michael Joseph. 2000. "Judgment and Truth in Frege". Journal of the History of Philosophy 38 (4): 549-81. https://doi.org/10.1353/hph.2005.0032

Künne, Wolfgang. 2008. "Frege on Truths, Truth and the True". Studia Philosophica Estonica 1 (1): 5-42.

Künne, Wolfgang. 2003. Conceptions of Truth. Oxford: Oxford University Press.

Oliver, Alex, and Timothy Smiley. 2016. Plural Logic. Oxford: Oxford University Press.

Ricketts, Thomas G. 1986. "Objectivity and Objecthood: Frege's Metaphysics of Judgment". In Frege Synthesized: Essays on the Philosophical and Foundational Work of Gottlob Frege, edited by Leila Haaparanta, and Jaakko Hintikka, 65-96. Dordrecht, The Netherlands: D. Reidel Publishing Company.

Schantz, Richard. 1998. "Was Tarski a Deflationist?". Logic and Logical Philosophy 6: 157-72. 
Smith, Nicholas J. J. 2009. "Frege's Judgement Stroke and the Conception of Logic as the Study of Inference not Consequence". Philosophy Compass 4: 639 65. https://doi.org/10.1111/j.1747-9991.2009.00219.x

Wright, Crispin. 1998. "Truth: A Traditional Debate Reviewed." Canadian Journal of Philosophy 24 (1): 31-74.

https://doi.org/10.1080/00455091.1998.10717495 